\title{
El maltrato escolar en centros de educación primaria en la provincia de Huelva (España)
}

\author{
Sara Conde Vélez ${ }^{1}$ y José Antonio Ávila Fernández ${ }^{2}$
}

\begin{abstract}
RESUMEN
El trabajo que se propone versa fundamentalmente sobre la percepción que tienen los niños y niñas sobre el maltrato entre compañeros y compañeras en la Educación Primaria así como el conocimiento de las intervenciones que realizan los centros educativos para prevenirla. En este sentido se procura un acercamiento al estudio de un contexto educativo, en el que el profesorado muestra su preocupación por los problemas de interrelación que se producen en él. Para ello se diseña y aplica un cuestionario a una muestra de 2156 sujetos, pertenecientes a treinta centros de Educación Infantil y Primaria de la provincia de Huelva. Los resultados han permitido destacar la presencia, en primer lugar, del maltrato verbal, tanto directo como indirecto, seguido de la exclusión social y el maltrato físico directo. El alumnado de Educación Primaria se define, generalmente, como maltratador ocasional, aunque es baja su apreciación en los valores moderado y grave.
\end{abstract}

Palabras Clave: bullying, escuela, violencia, primaria.

\section{School Abuse in Primary Schools in the Province of Huelva (Spain)}

\begin{abstract}
The work proposed is fundamentally about the perception that children have about the abuse between classmates in Primary Education as well as the knowledge of the interventions carried out by educational centers to prevent it. In this respect an approach to the study seeks an educational context, in which the faculty is concerned about the problems of interaction that occur in it. For it is designed and applied a questionnaire to a sample of 2,156 subjects, belonging to thirty Primary Education centers in the province of Huelva. The results have highlighted the presence, first, of both direct and indirect abuse, followed by social exclusion and direct physical and verbal abuse. The students of Primary Education are defined generally as occasional abusers, still low for its finding in moderate to severe values.
\end{abstract}

Keywords: bullying, school, violence, primary school.

Indicaba el Defensor del Pueblo (2000):

La violencia, execrable siempre y sea cual sea la razón que pretende justificarla, tiene un grado mayor de perversidad cuando afecta a niños y jóvenes. Cualquier niño, víctima o testigo de un acto violento, no solo padece las consecuencias inmediatas de este, sino que además incorpora a

1 y 2 Universidad de Huelva, España; sara.conde@dedu.uhu.es 
su desarrollo personal una experiencia negativa de consecuencias impredecibles en el futuro (p. 9).

Esta cita que precede nos sitúa, indefectiblemente, en la preocupación de las más altas instituciones por los problemas que se producen en el ámbito de la convivencia en los recintos escolares, que no afectan únicamente a los autores directos de los mismos, sino también a cuantos a su alrededor se encuentran como observadores, considerados como sujetos implicados en las acciones de acoso escolar.

Ovejero, Smith y Yubero (2013) construyen la definición de acoso escolar recopilando información de varios autores. Indican que es una violencia prolongada (mental y/o fisica), llevada a cabo por un individuo o grupo y dirigida contra un individuo que no es capaz de defenderse. Es una conducta prolongada en el tiempo y sistemática, que produce una relación de desequilibrio de poder entre agresores y víctimas. Presenta cuatro rasgos esenciales que la caracterizan:

- Ser una conducta violenta.

- Ser un comportamiento intencional.

- Repetición en el tiempo de tales conductas.

- Desequilibrio de poder y/o fuerza entre el agresor/a y su víctima, donde es muy frecuente la exclusión y/o el rechazo social (Wang, Iannotti, \& Nansel, 2009).

Las definiciones relacionadas con el acoso escolar son numerosas y su significado y alcance son diferentes según el país de referencia, por lo que los conceptos empleados no son siempre equivalentes.

Olweus (2006) lo identifica con acciones intimidatorias en las que se producen agresiones físicas, verbales y psicológicas. Albaladejo-Blázquez, Ferrer-Cascales, Reig-Ferrer y Fernández-Pascual (2013) entienden por acoso escolar que una persona sea agredida fisicamente, insultada, socialmente excluida, amenazada o atemorizada por otros/as de forma puntual y no reiterada (Ortega, del Rey, \& Mora-Merchán, 2001). Se caracteriza por ser una acción intencional deliberadamente encauzada a provocar daño de baja o moderada intensidad, la cual se lleva a cabo en contextos escolares, ya sea dirigida al alumnado, al profesorado o a los objetos propios del entorno educativo.

Marín-Martínez y Reidl-Martínez (2013) definen el acoso escolar como una conducta realizada con la intención de hacer daño. A veces en las relaciones de amistad entre los alumnos surgen comportamientos que pueden considerarse como agresivos, pero no tiene por qué ser acoso escolar. No necesariamente un comportamiento agresivo se realiza con la intención de provocar un daño.

Farrington (2005) incluye un elemento más en la definición de acoso escolar, el desequilibrio físico o psicológico de poder. La inclusión de este término hace desaparecer la igualdad en la conducta, y muestra que existe dominación del agresor y sumisión de la víctima.

En definitiva, e incluyendo todos los términos que designan los problemas de interacción entre el alumnado, los autores de esta investigación proponen la siguiente definición: Es un fenómeno escolar que se produce entre el alumnado, en el que se pone de manifiesto un comportamiento contrario a las normas de convivencia, individualmente o en grupo, públicamente o en privado, y en el que los sujetos agresores exhiben su poder sobre las víctimas. 
Para concluir con este apartado se expone de manera resumida las principales aportaciones destacadas por los autores:

- Acto intencional.

- Se lleva a cabo de forma sistemática.

- Desequilibrio de poder entre agresor/víctima.

- Conducta violenta: física, verbal, psicológica.

- Individual o grupal.

\section{Tipos de acoso escolar}

En una cuestión en la que existe dificultad en la definición, como consecuencia, se encuentra dificultad para enumerar su tipología. Es cierto que se habla de intensidad y duración, de direccionalidad, del sector implicado, pero las aportaciones sobre las diferentes situaciones violentas entre iguales o no se acometen, o no se definen, o no se diferencian con exactitud; en muchas ocasiones definición y tipología no concuerdan.

Carbonell (2009) comenta que el acoso escolar se puede presentar de diversas maneras:

- Físico: dando empujones, patadas, realizando agresiones con objetos.

- Verbal: profiriendo insultos y motes, menospreciando en público, realizando burlas sobre defectos físicos.

- Psicológico: minando la autoestima y fomentando la sensación de temor de la víctima.

- Social: excluyendo o aislando, rehuyendo la mirada, no contestando, impidiendo la participación, coaccionando a los amigos, no estando a su lado.

- Sexual: tocamientos sin consentimiento y acoso verbal de contenido sexual.

- Racial: relacionado con el origen étnico o creencias.

- Acoso escolar virtual, electrónico, digital: mediante el uso de las tecnologias y las redes sociales.

Rodicio-García e Iglesias-Cortizas (2011) proponen una clasificación de acoso atendiendo a cuatro criterios: la forma en que se produce, el modo de realizarlo, los actores intervinientes y el medio por el que se realiza.

En la investigación de Martínez (2012) se amplian las conductas referidas al tipo ciberbullying y se aplican en toda su extensión a ambos niveles educativos (Primario y Secundario).

El ciberbullying o ciberacoso se define como un comportamiento en línea difamatorio a través del uso de tecnologías de la información y la comunicación, como puede ser el correo electrónico, mensajes al teléfono móvil, mensajería instantánea. Este comportamiento procede de un individuo o un grupo que, deliberadamente y de forma repetitiva y hostil, pretende dañar a otro (Belsey, 2005). Según Arón (2008) el ciberbullying también es conocido como ciberacoso escolar. En él, los menores trasladan a internet sus insultos y amenazas y hacen pública la identidad de la víctima en un foro determinado (blogs, websites), incluso facilitan en algunos casos sus teléfonos, de manera que gente extraña se puede adherir a la agresión.

\section{Sujetos implicados en el acoso escolar}


El estudio de los sujetos intervinientes en el acoso escolar nos sitúa en la clasificación que la mayoría de los autores hacen al respecto: agresor, víctima y observador.

De acuerdo con lo propuesto por Quintana, Montgomery, Malaver y Ruíz (2010), los protagonistas del acoso escolar son:

- El agresor suele ser un individuo con fortaleza física mayor, con falta de empatía afectiva, sin sentimientos de culpa... Según Arroyave (2012) son dominantes, impulsivos y reaccionan fácilmente al conflicto, al interpretar cosas sin significado como agresivas.

- La víctima acostumbra a ser de menor fortaleza fisica, puede pertenecer a minorias étnicas o sociales, tiene una baja autoestima. No obstante Díaz-Aguado, Martínez-Arias y Martín-Babarro (2013) apuntan que las características de las víctimas del acoso están cambiando: dejan de centrarse en carencias psicológicas individuales para explicarlas en relación con el contexto en el que se produce.

- Los espectadores identifican víctimas y agresores, incluso algunos de ellos muestran una actitud complaciente ante la inequidad mientras que otros son indiferentes y/o toleran estas situaciones injustas.

Con respecto a los sujetos implicados en el acoso escolar, Ovejero et al. (2013) propone cuatro tipos de protagonistas del maltrato escolar: el maltratador escolar, la víctima, los colaboradores y los testigos indiferentes.

Es intención de este trabajo procurar un acercamiento al estudio de un contexto educativo en el que el profesorado muestra su inquietud por los problemas de interrelación entre el alumnado. Por ello, el objetivo general de este estudio es valorar la percepción que tienen los niños y niñas sobre el maltrato entre compañeros y compañeras en los centros de Educación Primaria en la provincia de Huelva (España), que se puede desglosar en los siguientes objetivos especificos:

- Valorar la percepción que tienen los niños/as sobre el acoso escolar en los centros de Educación Primaria en la provincia de Huelva (España).

- Dar cuenta, desde la opinión del alumnado, de las intervenciones que se llevan a cabo en los centros escolares para solucionar los problemas de acoso escolar.

\section{MÉTODO}

\section{Participantes}

La población objeto de estudio es el alumnado de educación primaria; el tamaño de la muestra asciende a 2156 sujetos, pertenecientes a treinta centros de Educación Infantil y Primaria de la provincia de Huelva (España), correspondientes a las seis comarcas en que esta se divide y se distribuyen en veinticuatro localidades. Para la selección de la muestra se ha utilizado un muestreo incidental en los que se utilizan los elementos de la población más fácilmente accesibles. Con respecto al género, el $51.40 \%$ del alumnado son niños y el 48.60\% son niñas. Las edades están comprendidas entre 8 y 13 años $(M=10.05 ; D T=1.31 ; \operatorname{Mod}=10 ; \operatorname{Med}=10)$.

Es necesario contemplar, en todo momento, que la muestra de alumnado participante en la investigación se corresponde con aquéllos que cursan segundo y tercer ciclo de Educación Primaria, como se viene haciendo en otros estudios similares (Albaladejo-Blázquez et al., 2013; Ortega, del Rey, \& Mora- 
Merchán, 2008). El alumnado participante en la investigación se distribuye de la siguiente forma: los que cursan tercero (532), cuarto (574), quinto (532) y sexto (518) curso de Educación Primaria.

Tabla 1.

Alumnado de segundo y tercer ciclos. Provincia de Huelva.

\begin{tabular}{lccc}
\hline Curso & Niños & Niñas & TOTAL \\
\hline Tercero & 2802 & 2646 & 5448 \\
Cuarto & 2965 & 2823 & 5788 \\
Quinto & 2732 & 2592 & 5324 \\
Sexto & 2968 & 2728 & 5696 \\
\hline Totales & 11467 & 10789 & 22256 \\
\hline
\end{tabular}

Fuente: Consejería de Educación de la Junta de Andalucía

La tabla 1 da cuenta de los datos de la población a la que se dirige la investigación, suponiendo la muestra un 9.69\% del total de la población (22.256), cantidad suficiente para el acercamiento a la generalización de los datos obtenidos.

\section{Instrumento de recogida de datos}

Se diseña un cuestionario para el cual se ha realizado un extenso estudio de propuestas de cuestionarios de diversa autoría (Avilés-Martínez, 1999; Collell \& Escudé, 2003; Fernández \& Ortega, 1998; Gómez Bahillo, 2006; Jiménez, 2007; Lera \& Olias, 2002; Ortega et al., 2008).

Se procedió a presentar una primera propuesta a expertos de la investigación, la orientación y el magisterio, para su validación. Las sugerencias y observaciones de los expertos sobre el instrumento de investigación sirvieron para realizar las modificaciones oportunas que más tarde se pasaron a la muestra piloto. Realizado el estudio pertinente de las reflexiones aportadas por estos, se formalizó el Cuestionario para la Detección del Acoso Escolar en la Escuela Primaria (ver tabla 4), que, en un primer momento, se pasó a una muestra piloto compuesta por alumnado de las edades a las que se dirigia la investigación (24 sujetos). El estudio se centraba en analizar la consistencia interna del constructo (escala de medida) sobre la fiabilidad, para ello se calculó el Alfa de Cronbach y se obtuvo un .83, lo que supera el valor recomendable (.70) para esta propiedad. La muestra piloto supuso la comprobación de la comprensión de cada apartado, el ritmo de ejecución y la necesidad de apoyo para centrar algunos contenidos. En la tabla 2 se detalla la procedencia de los elementos de la muestra.

Tabla 2.

Muestra piloto.

\begin{tabular}{lccc}
\hline \multirow{2}{*}{ Nivel } & \multicolumn{2}{c}{ Género } & \multirow{2}{*}{$\begin{array}{c}\text { Total } \\
\text { alumnado }\end{array}$} \\
\cline { 2 - 3 } Tercero & Niños & Niñas & \\
\cline { 2 - 3 } Cuarto & 4 & 2 & 6 \\
Quinto & 3 & 3 & 6 \\
Sexto & 2 & 4 & 6 \\
\hline Totales & 3 & 3 & 6 \\
\hline
\end{tabular}


Tras el estudio de los cuestionarios y la posterior simplificación de categorías, se determina la siguiente tabla de dimensiones (tabla 3), que regirá el proceso de la presente investigación.

En ella se incluyen las definiciones de las dimensiones que se van a estudiar, acotando, así, el contenido de las mismas.

Tabla 3.

Definición de dimensiones.

\begin{tabular}{|c|c|}
\hline Dimensiones & Definiciones \\
\hline Variables sociodemográficas & $\begin{array}{l}\text { Incorporará este apartado aquellos datos relativos a las variables } \\
\text { que describan las características del niño o niña que participa en la } \\
\text { muestra, como son: localidad, centro, edad, curso, sexo, } \\
\text { nacionalidad, y algún indicador familiar. Se mantendrá el anonimato } \\
\text { para cuidar la confidencialidad de los participantes. }\end{array}$ \\
\hline $\begin{array}{l}\text { Lugares en los que se produce el } \\
\text { maltrato }\end{array}$ & $\begin{array}{l}\text { Se establece como espacio todos los posibles en el edificio escolar, } \\
\text { incluidos aquéllos que son comunes. Además se estimará como } \\
\text { espacio los externos al centro, siempre que el suceso se produzca } \\
\text { por un problema escolar. }\end{array}$ \\
\hline Sujetos intervinientes & $\begin{array}{l}\text { Se consideran como sujetos implicados en el maltrato entre iguales } \\
\text { a los agresores (autores del acoso a un igual), las víctimas } \\
\text { (receptores/as de la agresión) y los espectadores (los compañeros } \\
\text { y compañeras que observan los hechos). }\end{array}$ \\
\hline Intervenciones & $\begin{array}{l}\text { Se entenderá como intervenciones aquellas acciones que se } \\
\text { realizan para formar en la convivencia, evitar o solucionar los casos } \\
\text { de acoso que se producen en el contexto escolar, ya sean acciones } \\
\text { del alumnado como del profesorado y que pueden realizarse antes, } \\
\text { durante o después de los hechos intimidatorios. }\end{array}$ \\
\hline Formas de maltrato & $\begin{array}{l}\text { Se utiliza el esquema más simple de formas establecidas de } \\
\text { maltrato en estudios relevantes: maltratos físico, verbal y social, } \\
\text { aunque a la hora de establecer las cuestiones correspondientes a } \\
\text { este aspecto se alude a diferentes acciones consideradas en cada } \\
\text { una de estas tres formas generales. }\end{array}$ \\
\hline Relaciones & $\begin{array}{l}\text { Se pretende realizar preguntas cuyas respuestas expresen la } \\
\text { valoración que niños y niñas hacen del trato con sus compañeros y } \\
\text { compañeras y con el profesorado. }\end{array}$ \\
\hline
\end{tabular}

Antes de la aplicación del cuestionario, el encuestador centró al alumnado en la tarea y facilitó explicaciones sobre la definición de acoso escolar. Proporcionó instrucciones para que su aplicación se realizara de forma igualitaria a todos los grupos en los que se administró: motivación ante la tarea, apoyo en su realización, reflexión e información sobre su contenido y que el grupo de alumnos/as que lo cumplimentaran siguieran el mismo ritmo. 
Tabla 4.

Cuestionario para la detección del Acoso Escolar en Educación Primaria.

CUESTIONARIO PARA LA DETECCIÓN DEL ACOSO ESCOLAR EN EDUCACIÓN PRIMARIA

\section{VARIABLES SOCIODEMOGRÁFICAS}

1. COMARCAS:

2. PUEBLO O CIUDAD:

3. COLEGIO:

4. EDAD:

5. CURSO:

6. SEXO

Niño

Niña

7. NACIONALIDAD:

8. ¿Con quién vives?

a) Con mis padres

b) Sólo con uno de ellos

c) Con otros familiares

d) Con otra familia

e) En una residencia

\section{RELACIONES}

9. Las relaciones tuyas con los compañeros/as son

10. Las relaciones tuyas con tu tutor/a son

11. Las relaciones tuyas con los demás maestros/as son

12. Las relaciones entre los compañeros/as de la clase son

\begin{tabular}{|c|c|c|c|}
\hline $\begin{array}{c}\text { Muy } \\
\text { buenas }\end{array}$ & Buenas & Malas & $\begin{array}{c}\text { Muy } \\
\text { malas }\end{array}$ \\
\hline & & & \\
\hline & & & \\
\hline & & & \\
\hline $\begin{array}{l}\text { Muy } \\
\text { bien }\end{array}$ & Bien & Mal & Muy mal \\
\hline & & & \\
\hline
\end{tabular}

13. ¿Cómo te sientes tú cuando estás en el colegio?

14. ¿Cómo crees que es el ambiente del colegio?

\section{VÍCTIMA}

15. Me amenazan

16. Me pegan (golpes, patadas, puñetazos, zancadillas)

17. Me esconden mis cosas

18. Me rompen queriendo mis materiales (la mochila, los cuadernos)

19. Me quitan cosas

20. Me insultan, se meten conmigo, se burlan o ríen de mí

21. Me dicen motes para ofenderme

22. Hablan mal de mí

23. Me dejan solo/a, no se juntan conmigo para hacerme daño

24. No me dejan participar en el juego, no quieren jugar conmigo

25. No quieren estudiar conmigo para rechazarme

26. Me obligan a hacer algo que yo no quiero

\begin{tabular}{|c|c|c|c|}
\hline Nunca & $\begin{array}{c}\text { Alguna } \\
\text { vez }\end{array}$ & $\begin{array}{c}\text { Muchas } \\
\text { veces }\end{array}$ & Siempre \\
\hline & & & \\
\hline & & & \\
\hline & & & \\
\hline & & & \\
\hline & & & \\
\hline & & & \\
\hline & & & \\
\hline & & & \\
\hline & & & \\
\hline & & & \\
\hline & & & \\
\hline & & & \\
\hline
\end{tabular}

27. He sufrido otro tipo de acoso ¿Cuál? 
Tabla 4.

Cuestionario para la detección del Acoso Escolar en Educación Primaria (continuación).

\section{AGRESOR/A}

28. ¿Tú amenazas a algún compañero/a?

29. ¿Tú pegas a algún niño/a? (dando golpes, patadas puñetazos, zancadillas)

30. ¿Tú escondes cosas a tus compañeros/as?

31. ¿Tú rompes queriendo los materiales de los demás? (mochilas, cuadernos, libros...)

32. ¿Tú quitas cosas a tus compañeros/as?

33. ¿Tú insultas a los niños/as, te metes con ellos/as, te burla o te ríes?

34. ¿Tú dices motes a tus compañeros/as para ofenderlos?

35. ¿Tú hablas mal de tus compañeros/as?

36. ¿Tú dejas solos/as a algunos niños/as, no te juntas con ellos/as para hacerles daño?

37. ¿Tú no dejas participar en el juego a algún niño/a? ¿No quieres jugar con ellos/as?

38. ¿Tú no quieres estudiar con algún niño/a para rechazarlo/a?

39. ¿Tú obligas a algunos niños/as a hacer algo que ellos/as no quieren?

40. ¿Tú has realizado otro tipo de acoso? ¿Cuál?

Nunca $\begin{gathered}\text { Alguna Muchas } \\
\text { vez }\end{gathered}$
\begin{tabular}{|l|l|l|l|}
\hline & veces & Siempre \\
\hline & & & \\
\hline & & & \\
\hline & & & \\
\hline & & & \\
\hline & & & \\
\hline & & & \\
\hline & & & \\
\hline & & & \\
\hline & & & \\
\hline & & & \\
\hline & & & \\
\hline & & & \\
\hline & & & \\
\hline
\end{tabular}

\section{OBSERVADOR/A}

41. He visto amenazar a un compañero/a

42. He visto pegar a los demás (dar golpes, patadas puñetazos)

43. He visto esconder cosas a los compañeros/as

44. He visto romper queriendo los materiales a los compañeros/as

45. He visto quitar cosas a los compañeros/as

46. He oído insultar a compañeros/as, meterse con ellos, burlarse o reírse de ellos/as

47. He oído decir motes a los demás para ofenderlos

48. He oído hablar mal de compañeros/as

49. He visto dejar solos/as a compañeros/as, no juntarse con ellos/as para hacerles daño

50. He visto no dejar participar en el juego, no queriendo jugar con ellos/as

51. He visto no querer estudiar con algunos compañeros/as para rechazarlos

52. He visto obligar a otros/as a hacer algo que no querían hacer

53. He visto sufrir algún otro tipo de acoso a algún compañero/a

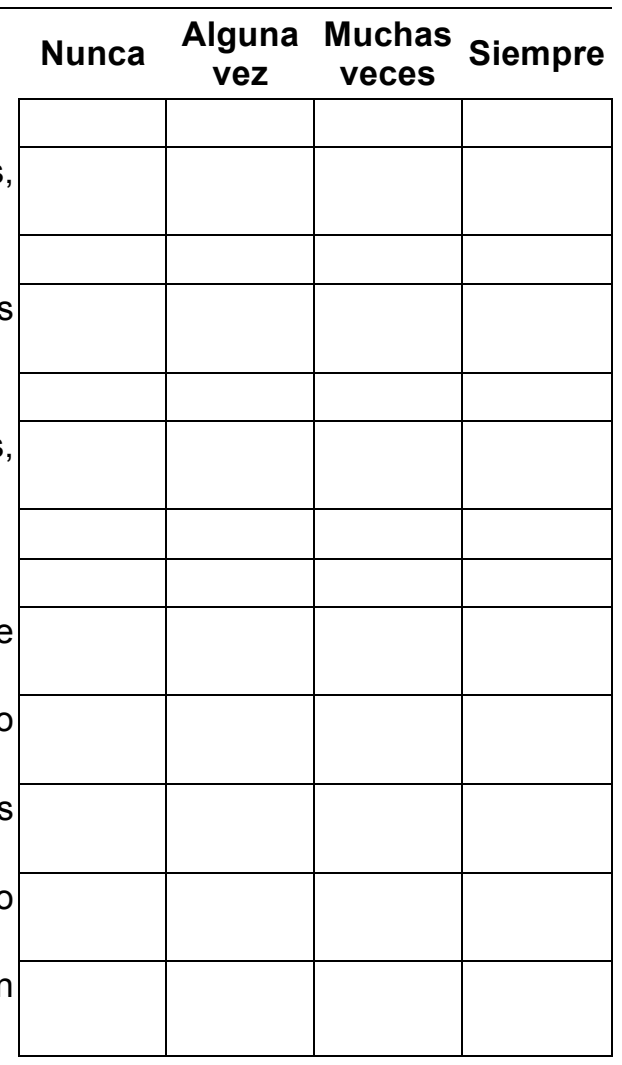


Tabla 4.

Cuestionario para la detección del Acoso Escolar en Educación Primaria (continuación).

\section{LUGARES EN LOS QUE SE PRODUCE EL MALTRATO}

54. ¿En qué lugar del colegio ocurren más veces los problemas de acoso escolar entre los compañeros/as?
a) En las entradas y salidas del colegio
b) En la clase, cuando está el tutor/a
c) En clase, cuando está otro maestro
d) En clase, cuando estamos solos, al cambiar el
e) En los pasillos maestro/a de grupo
g) En los recreos
f) En los baños
h) En otros lugares. Escribe cuál:

\section{QUIÉN ACOSA}

55. ¿Quién acosa más veces a los niños/as del colegio?
a) Un niño
b) Una niña
c) Varios niños
d) Varias niñas
e) Niños y niñas

\section{DÓNDE SE ENCUENTRA}

56. ¿Dónde se encuentran los acosadores?
a) En una clase de niños/as más pequeños
b) En mi clase
c) En una clase del mismo nivel
d) En una clase de niños mayores que yo

\section{INTERVENCIONES}

57. Cuando hay un problema de acoso en tu colegio ¿qué se hace?

a) Hay niños/as mediadores

b) Se dan charlas sobre convivencia

c) En las clases/en las asambleas tratamos temas de convivencia con los maestros

d) Los maestros/as nos llaman la atención

e) Los maestros/as nos hacen reflexionar en la clase y nos aconsejan

f) Los maestros/as castigan

g) Los maestros/as "ponen partes"

h) Los maestros/as mandan a los alumnos/as al Director/a o al Jefe/a de Estudios

i) Los niños/as ayudamos a las víctimas

j) Los niños/as separamos las peleas

k) Los niños/as reñimos a los agresores

l) Los niños/as avisamos a los maestros/as

$\mathrm{m})$ Me meto también en la pelea

n) Los maestros/as llaman a los padres

ñ) Se expulsa a los niños/as que son acosadores/as

o) No se hace nada

p) Otras: (escribe cuáles)

Fuente: elaboración propia

\section{Procedimiento y análisis de datos}

La aplicación del cuestionario elaborado a la muestra provincial supuso crear una red de compromisarios con garantías, basada en los profesionales de los equipos de orientación.

Para el análisis se ha utilizado el programa informático SPSS.v17. Los datos se han estructurado en diferentes bloques para facilitar su lectura. 
De las características de la muestra, su distribución por sexo se reparte de forma equilibrada: el $51.39 \%$ (niños) frente a un $48.61 \%$ (niñas). La distribución de los sujetos participantes por curso queda establecida de la siguiente forma: $24 \%$ (sexto curso de primaria); $24.70 \%$ (tercer curso de primaria); $24 \%$ (quinto curso de primaria); y $26.60 \%$ (cuarto curso de primaria).

Respecto a la edad, téngase en cuenta que el alumnado de 13 años es alumnado de permanencia de un año más en algún ciclo escolar y que el alumnado de 9, 10 y 11 años comparte los cursos anterior y posterior, por razones de mes de nacimiento o permanencia de un año más en el ciclo. Su distribución queda conformada del siguiente modo: el $2.10 \%$ de la muestra tienen 13 años, el $13.10 \%$ tienen 12 años, el $14 \%$ tienen 8 años, el $22.80 \%$ tienen 9 años, el $23.40 \% 11$ años y el $24.70 \% 10$ años.

Seguidamente se describe la distribución de los participantes por comarca según su contribución a la muestra general. Distintas situaciones marcan esta participación, sobre todo las geográficas y poblacionales. La concentración de la población en la capital y su entorno, como en la costa y en el condado y la dispersión de los habitantes de la sierra, la mina y el Andévalo: Sierra (11.87\%); Andévalo (4.80\%); Costa (18.58\%); Cinturón (33.72\%); Condado (20.87\%); y mina (10.16\%).

Respecto a la nacionalidad, el $89.90 \%$ de la muestra es de nacionalidad española frente a un $10.10 \%$ que son inmigrantes.

Al explorar la situación del alumnado en el aspecto familiar que se solicita en la investigación con la cuestión “¿con quién vives?” se establece que un $11.80 \%$ del alumnado participante pertenece a familias monoparentales, cantidad que deja constancia de la rápida difusión de nuevos modelos familiares de nuestra sociedad.

Otros datos considerados en esta cuestión son los que definen al alumnado que vive con otros familiares $(1.25 \%)$, con otra familia $(0.23 \%)$ o en instituciones de acogida $(0.50 \%)$.

\section{RESULTADOS}

\section{Perspectiva de las relaciones en el centro}

La actividad relacional personal se plantea en razón a la convivencia con los compañeros y con el profesorado.

En correspondencia con las relaciones entre los compañeros destacan los valores muy buenas y buenas, que significan $97.69 \%$ del total de la muestra. No obstante en la investigación preocupan los datos cuyos resultados se asocian a connotaciones negativas a fin de intervenir en la solución de los problemas que muestran. De estos últimos se obtienen como malas el $1.99 \%$ y muy malas el $0.32 \%$.

Dos cuestiones tratan de obtener datos relativos a las relaciones del alumnado con el profesorado. En primer lugar las que se tienen con el tutor/a. Se alcanza la cantidad de $2.13 \%$ en los valores malas y muy malas, mientras que en las relaciones con los demás docentes, los mismos valores suman 1.72 $\%$. En los datos, las diferencias se establecen a favor de la tutoría; así en cuanto a muy buenas las cantidades son $66.93 \%$ frente a $49.40 \%$, y en el apartado buenas las diferencias anteriores se alternan (30.94 \% y $48.89 \%)$. 
Una vez solicitada la información de relaciones desde la óptica personal, se presenta, a continuación, la visión que tiene el alumnado sobre las relaciones entre compañeros y compañeras.

Las diferencias son evidentes cuando se cambia la dirección de la cuestión. Las relaciones entre compañeros se observan como más problemáticas que las relaciones propias con los demás. Aunque de manera global los resultados se pueden considerar óptimos $(49.95 \%$ en la consideración de las relaciones como muy buenas y $44.43 \%$ como buenas), los datos de relaciones negativas se amplian hasta un $4.78 \%$ en la percepción de malas y existe un $0.83 \%$ que las aprecian como muy malas.

Dos preguntas pretenden obtener datos sobre la ambientación global del centro, desde la consideración personal como elemento integrado en el grupo social y desde la observación de los demás compañeros. Esta estimación sobre el ambiente del centro desde las dos perspectivas, personal y ajena, acrecientan los datos asociados a connotaciones negativas desde la visión de las relaciones, que arrojan un $3.06 \%$ y un $1.02 \%$ (malas, muy malas) en la primera opción y un $5.24 \%$ y un $1.25 \%$, en cuanto al ambiente general.

\section{Sujetos implicados en el acoso escolar}

Estos resultados se expresan iniciando su exposición con una observación de tipo global, construida con la simplificación del conjunto de los datos recabados en las cuestiones relacionadas con cada uno de los sujetos, y continúa, tras la inclusión de una recopilación de los obtenidos de cada tipo de sujeto, con una exposición descriptiva siguiendo la propuesta de tipos de maltrato del Defensor del Pueblo (2000). En el estudio que se ha aludido se establecía como formas de maltrato las que se indican a continuación:

- Maltrato físico (directo, indirecto).

- Maltrato verbal (directo, indirecto).

- Exclusión social.

- Maltrato mixto.

En la tabla 5 se deja constancia de la distribución de ítems, según el esquema anterior.

Tabla 5.

Correspondencia de ítems y tipos de maltrato.

\begin{tabular}{ll}
\hline TIPO DE MALTRATO & ÍTEMS RELACIONADOS \\
\hline Maltrato físico directo & 2 \\
Maltrato físico indirecto & $3,4,5$ \\
Maltrato verbal directo & 6,7 \\
Maltrato verbal indirecto & 8 \\
Exclusión social & $9,10,11$ \\
Maltrato mixto & 1,12 \\
\hline
\end{tabular}

Fuente: elaboración propia

Por otra parte, se ha establecido la intensidad de las acciones de maltrato en relación con los valores del cuestionario. Así, se considerará el valor alguna vez como situación leve, muchas veces como moderada y siempre como grave. 


\section{Datos desde la posición de víctima}

Se deduce del compendio de datos obtenidos en los ítems relacionados con la victimización, que un $29.63 \%$ del alumnado percibe su posicionamiento como sujeto agredido por sus compañeros/as alguna vez, un $5.12 \%$ muchas veces y un $2.26 \%$ siempre. La ausencia de victimización es sentida por el $62.99 \%$ de los encuestados.

Tabla 6.

Recopilación de resultados en porcentajes de la dimensión "sujetos intervinientes: víctima".

\begin{tabular}{|c|c|c|c|c|}
\hline Ítems & Nunca & $\begin{array}{l}\text { Alguna } \\
\text { Vez }\end{array}$ & $\begin{array}{l}\text { Muchas } \\
\text { Veces }\end{array}$ & Siempre \\
\hline Me amenazan & $53.57 \%$ & $41.56 \%$ & $4.04 \%$ & $0.83 \%$ \\
\hline $\begin{array}{l}\text { Me pegan (golpes, patadas, puñetazos, } \\
\text { zancadillas) }\end{array}$ & $51.62 \%$ & $41.33 \%$ & $5.47 \%$ & $1.58 \%$ \\
\hline Me esconden mis cosas & $59.23 \%$ & $35.25 \%$ & $4.27 \%$ & $1.25 \%$ \\
\hline $\begin{array}{l}\text { Me rompen queriendo mis materiales (la } \\
\text { mochila, los cuadernos, los libros...) }\end{array}$ & $87.94 \%$ & $10.58 \%$ & $0.97 \%$ & $0.51 \%$ \\
\hline Me quitan cosas & $60.39 \%$ & $33.86 \%$ & $4.68 \%$ & $1.07 \%$ \\
\hline $\begin{array}{l}\text { Me insultan, se meten conmigo, se burlan o } \\
\text { ríen de mí }\end{array}$ & $39.94 \%$ & $43.00 \%$ & $11.60 \%$ & $5.47 \%$ \\
\hline $\begin{array}{l}\text { Me dicen motes para ofenderme } \\
\text { Hablan mal de mí }\end{array}$ & $\begin{array}{l}52.74 \% \\
45.64 \%\end{array}$ & $\begin{array}{l}31.31 \% \\
43.09 \%\end{array}$ & $\begin{array}{l}9.42 \% \\
7.88 \%\end{array}$ & $\begin{array}{l}6.54 \% \\
3.39 \%\end{array}$ \\
\hline $\begin{array}{l}\text { Me dejan solo/a, no se juntan conmigo } \\
\text { para hacerme daño }\end{array}$ & $73.65 \%$ & $19.94 \%$ & $4.31 \%$ & $2.09 \%$ \\
\hline $\begin{array}{l}\text { No me dejan participar en el juego, no } \\
\text { quieren jugar conmigo }\end{array}$ & $71.94 \%$ & $23.05 \%$ & $3.34 \%$ & $1.67 \%$ \\
\hline $\begin{array}{l}\text { No quieren estudiar conmigo para } \\
\text { rechazarme }\end{array}$ & $87.24 \%$ & $9.37 \%$ & $1.95 \%$ & $1.44 \%$ \\
\hline Me obligan a hacer algo que yo no quiero & $70.92 \%$ & $23.79 \%$ & $3.76 \%$ & $1.53 \%$ \\
\hline
\end{tabular}

Fuente: elaboración propia

En estas cantidades destacan por ausencia del fenómeno el maltrato físico indirecto $(59.23 \%, 87.94 \%$ y $60.39 \%)$ y la exclusión social $(73.65 \%$, $71.94 \%$ y $87.24 \%)$. Como valores que denotan más presencia: el insulto $(43.00 \%)$, la maledicencia $(43.09 \%)$, las amenazas $(41.56 \%)$ y las agresiones físicas $(41.33 \%)$ en el apartado leve; el maltrato verbal, tanto directo como indirecto, en las opciones moderadas y graves $(11.60 \%$ y $5.47 \% ; 9.42 \%$ y $6.54 \% ; 7.88 \%$ y $3.39 \%$; tabla 6).

En resumen, entre los datos expuestos destaca la presencia de acoso grave por el uso de motes ofensivos, el insulto y la maledicencia (maltrato verbal); en segundo lugar aparece la exclusión social, el sentirse ignorados o rechazados, y, a continuación, el maltrato físico directo, el recibir golpes, patadas, puñetazos o zancadillas.

\section{Datos desde la posición de agresor}

La exposición de datos relacionados con los sujetos del maltrato continúa con los correspondientes desde el posicionamiento de los encuestados como agresores. Las doce cuestiones que solicitaban información desde la posición de víctima ahora se acomodan a la solicitud de datos como agresor.

De los datos generales sobre agresión, se desprende que el $19.07 \%$ del alumnado encuestado se autodefine como agresor ocasionalmente, el $1.03 \% \mathrm{y}$ el $0.50 \%$ sienten realizar acciones agresoras de manera moderada y grave. 
En la tabla 7 se exponen los datos obtenidos sobre agresión:

Tabla 7.

Recopilación de resultados en porcentajes de la dimensión "sujetos intervinientes: agresor".

\begin{tabular}{|c|c|c|c|c|}
\hline Ítems & Nunca & $\begin{array}{l}\text { Alguna } \\
\text { vez }\end{array}$ & $\begin{array}{l}\text { Muchas } \\
\text { veces }\end{array}$ & Siempre \\
\hline ¿Tú amenazas a algún compañero/a? & $75.09 \%$ & $23.75 \%$ & $0.79 \%$ & $0.37 \%$ \\
\hline $\begin{array}{l}\text { ¿Tú pegas a algún niño/a? (dando golpes, patadas, } \\
\text { puñetazos, zancadillas) }\end{array}$ & $65.82 \%$ & $31.96 \%$ & $1.53 \%$ & $0.70 \%$ \\
\hline ¿Tú escondes cosas a tus compañeros/as? & $79.78 \%$ & $19.16 \%$ & $0.74 \%$ & $0.32 \%$ \\
\hline $\begin{array}{l}\text { ¿Tú rompes queriendo los materiales de los demás? } \\
\text { (mochilas, cuadernos, libros...) }\end{array}$ & $97.63 \%$ & $1.99 \%$ & $0.28 \%$ & $0.09 \%$ \\
\hline ¿Tú quitas cosas a tus compañeros/as? & $92.12 \%$ & $7.42 \%$ & $0.32 \%$ & $0.14 \%$ \\
\hline $\begin{array}{l}\text { ¿Tú insultas a los niños/as, te metes con ellos/as, te } \\
\text { burlas o te ríes? }\end{array}$ & $59.23 \%$ & $38.17 \%$ & $1.95 \%$ & $0.65 \%$ \\
\hline $\begin{array}{l}\text { ¿Tú dices motes a tus compañeros/as para } \\
\text { ofenderlos? }\end{array}$ & $70.73 \%$ & $26.44 \%$ & $2.04 \%$ & $0.79 \%$ \\
\hline ¿Tú hablas mal de tus compañeros/as? & $67.44 \%$ & $30.84 \%$ & $1.35 \%$ & $0.37 \%$ \\
\hline $\begin{array}{l}\text { ¿Tú dejas solos/as a algunos niños/as, no te juntas } \\
\text { con ellos/as para hacerles daño? }\end{array}$ & $86.27 \%$ & $12.06 \%$ & $1.21 \%$ & $0.46 \%$ \\
\hline $\begin{array}{l}\text { ¿Tú no dejas participar en el juego a algún niño/a? } \\
\text { ¿No quieres jugar con ellos/as? }\end{array}$ & $77.74 \%$ & $20.08 \%$ & $1.07 \%$ & $1.11 \%$ \\
\hline $\begin{array}{l}\text { ¿Tú no quieres estudiar con algún niño/a para } \\
\text { rechazarlo/a? }\end{array}$ & $90.49 \%$ & $8.12 \%$ & $0.70 \%$ & $0.70 \%$ \\
\hline $\begin{array}{l}\text { ¿Tú obligas a algunos niños/as a hacer algo que } \\
\text { ellos/as no quieren? }\end{array}$ & $90.35 \%$ & $8.86 \%$ & $0.46 \%$ & $0.32 \%$ \\
\hline
\end{tabular}

Fuente: elaboración propia

En el posicionamiento como agresor se destacan los datos correspondientes a agresiones leves, relacionadas con el maltrato verbal directo, en primer lugar, seguido de maltrato físico directo y de maltrato verbal indirecto $(38.17 \%, 31.96 \%$ y $30.84 \%)$.

En cuanto al maltrato moderado, los datos que sobresalen están relacionados con el maltrato verbal, el maltrato físico y la exclusión social. Por último, en la intensidad grave, destaca la exclusión, el maltrato verbal y el maltrato fisico. Se analizan con más detalle estas observaciones generales en los comentarios que siguen.

En sintesis, los datos inferiores, desde la autopercepción del alumnado como agresor, se establecen en el maltrato físico indirecto, referido a romper los materiales ajenos y al robo, en el rechazo en el estudio y en obligar a hacer a los compañeros cosas que no quieren con amenazas; alguna vez se realizan destacadamente acciones relacionadas con el insulto, la agresión física directa y la maledicencia; aparecen muchas veces realizadas acciones de maltrato verbal, $y$, entre las situaciones de maltrato en su forma grave, asumidas por el agresor, destaca el rechazo en el juego, por encima de la agresión verbal, física y el rechazo en el estudio.

En referencia al actor de la agresión se solicita información para determinar el género, si la actividad es individual o colectiva y a la clase a la que pertenece.

Los resultados de este tema muestran que lo más habitual es encontrarse con niños acosadores que forman grupos; el $39.94 \%$ así lo 
indican. Le siguen en elección los grupos de niños y niñas (34.65\%) y, a continuación, un niño, que alcanza el $20.55 \%$. En menor medida son elegidas las niñas individualmente o en grupo, que consiguen el $1.76 \%$, cuando realizan la actividad agresora ellas solas, y el $3.11 \%$, en grupo.

En el apartado correspondiente a los acosadores se procura saber dónde se encuentran estos en el ámbito escolar.

Se expone, con estos datos, que es más frecuente que los agresores se encuentren en una clase de niños mayores que la víctima (64.47\%). La presencia de agresores de la misma edad de la víctima puede estar en el mismo grupo de alumnado o en un grupo paralelo; estos datos, $51.95 \%$ y $38.36 \%$, tienen que ser estimados cuidadosamente, pues encontramos centros de una sola línea (en cada nivel solo hay un grupo de alumnos) que no permite elegir la segunda opción. No es frecuente que el agresor provenga de una clase inferior; aun así se obtiene el $13.03 \%$ de las respuestas.

\section{Datos desde la posición de observadores}

Es en esta posición donde se consiguen los testimonios más acentuados sobre intimidación, dado que todo el alumnado participa de esta situación de privilegio como espectador en el ámbito escolar en el que está integrado.

Cuando en las anteriores respuestas, en posicionamientos de víctima y agresor, se habian obtenido el $62.99 \%$ y el $79.39 \%$ que indicaban ausencia de victimización, al solicitar la información de las opiniones del alumnado desde la perspectiva de espectador se alcanza la cifra de $34 \%$ de ausencia.

Por tanto, desde la observación se van a recibir cantidades muy superiores de la presencia de acoso escolar.

E1 $38 \%$ del alumnado indica que observa acciones de maltrato leve; las acciones moderadas se producen según un $18.20 \%$ y las situaciones de maltrato grave son percibidas por un $6 \%$, datos que se sitúan muy por encima de los conseguidos bajo las anteriores posiciones.

La tabla 8, que recopila los datos obtenidos en la posición de observador, y que se incluye a continuación, descubre como acciones menos afectadas en el maltrato entre iguales las relacionadas con el maltrato físico indirecto, las que tienen que ver con los materiales ajenos -"He visto esconder cosas a los compañeros", "He visto romper queriendo los materiales a los compañeros (mochilas, cuadernos, libros...)", "He visto quitar cosas a los compañeros" -, así como el rechazo en el estudio y las acciones para obligar a hacer lo que no se quiere.

Pero es notable el aumento de la percepción del alumnado en su actitud de observador en todas las situaciones de maltrato, que en el valor alguna vez es similar y supera casi siempre el $40 \%$. Entre estas destacan las amenazas "He visto amenazar a un compañero/a"-, todas las formas de maltrato físico "He visto pegar a los demás (dar golpes, patadas, puñetazos, poner zancadillas)"-, la maledicencia -"He oído hablar mal de compañeros/as"- y la exclusión en el juego -"He visto no dejar participar en el juego, no queriendo jugar con ellos/as"-. Solo baja de esta cantidad las situaciones obligar a hacer lo que no se quiere, el rechazo en el estudio y romper los materiales de los demás. 
Tabla 8.

Recopilación de resultados en porcentajes de la dimensión "sujetos intervinientes: observador".

\begin{tabular}{|c|c|c|c|c|}
\hline Ítems & Nunca & $\begin{array}{l}\text { Alguna } \\
\text { vez }\end{array}$ & $\begin{array}{l}\text { Muchas } \\
\text { veces }\end{array}$ & Siempre \\
\hline He visto amenazar a un compañero/a & $17.07 \%$ & $48.70 \%$ & $27.41 \%$ & $6.82 \%$ \\
\hline $\begin{array}{l}\text { He visto pegar a los demás (dar golpes, patadas, } \\
\text { puñetazos, poner zancadillas) }\end{array}$ & $11.92 \%$ & $47.68 \%$ & $33.16 \%$ & $7.24 \%$ \\
\hline He visto esconder cosas a los compañeros/as & $35.44 \%$ & $45.59 \%$ & $15.31 \%$ & $3.66 \%$ \\
\hline $\begin{array}{l}\text { He visto romper queriendo los materiales a los } \\
\text { compañeros/as (mochilas, cuadernos, libros...) }\end{array}$ & $65.07 \%$ & $26.07 \%$ & $6.31 \%$ & $2.55 \%$ \\
\hline He visto quitar cosas a los compañeros/as & $44.57 \%$ & $40.54 \%$ & $11.32 \%$ & $3.57 \%$ \\
\hline $\begin{array}{l}\text { He oído insultar a compañeros/as, meterse con } \\
\text { ellos, burlarse o reírse de ellos }\end{array}$ & $12.38 \%$ & $43.32 \%$ & $32.24 \%$ & $12.06 \%$ \\
\hline $\begin{array}{l}\text { He oído decir motes a los demás para ofenderlos } \\
\text { He oído hablar mal de compañeros/as }\end{array}$ & $\begin{array}{l}22.73 \% \\
22.54 \%\end{array}$ & $\begin{array}{l}40.68 \% \\
47.96 \%\end{array}$ & $\begin{array}{l}26.11 \% \\
22.54 \%\end{array}$ & $\begin{array}{l}10.48 \% \\
6.96 \%\end{array}$ \\
\hline $\begin{array}{l}\text { He visto dejar solos/as a compañeros/as, no } \\
\text { juntarse con ellos/as para hacerles daño }\end{array}$ & $32.70 \%$ & $43.14 \%$ & $19.11 \%$ & $5.06 \%$ \\
\hline $\begin{array}{l}\text { He visto no dejar participar en el juego, no } \\
\text { queriendo jugar con ellos/as }\end{array}$ & $29.08 \%$ & $47.63 \%$ & $18.69 \%$ & $4.59 \%$ \\
\hline $\begin{array}{l}\text { He visto no querer estudiar con algunos } \\
\text { compañeros/as para rechazarlos }\end{array}$ & $61.97 \%$ & $27.46 \%$ & $8.21 \%$ & $2.37 \%$ \\
\hline $\begin{array}{l}\text { He visto obligar a otros/as a hacer algo que no } \\
\text { querían hacer }\end{array}$ & $48.47 \%$ & $35.48 \%$ & $12.76 \%$ & $3.29 \%$ \\
\hline
\end{tabular}

Fuente: elaboración propia

En relación con las dos intensidades superiores, moderada y grave, "pegar a los demás" (33.16 \%) sigue tomando la delantera de las acciones agresivas, seguida del insulto (32.24\%), las amenazas a los compañeros $(27.41 \%)$ y decir motes para ofender $(26.11 \%)$ al describir las moderadas, y las acciones consideradas graves alcanzan el $12.06 \%$ y el $10.48 \%$ las relacionadas con el maltrato verbal directo (insultar y decir motes), el $7.24 \%$ el maltrato físico, el $6.96 \%$ el maltrato verbal indirecto (hablar mal de los demás) y el $6.82 \%$ las amenazas.

\section{Lugares donde se produce el acoso}

La demanda de información sobre los lugares donde se producen las acciones de maltrato entre iguales se realiza presentando una serie de espacios posibles y solicitando la elección de hasta tres opciones.

De ellas, las más elegidas, según se lee en la tabla siguiente, son:

- Los recreos (88.17\%).

- Las entradas y salidas (58.35\%).

- La clase, cuando están los alumnos/as solos, al cambiar el maestro/a de grupo (51.53\%).

Destaca "los recreos" sobre las demás opciones, como se aprecia en la tabla 9.

La demás elecciones presentan cantidades similares. Se hace notar que la opción menos puntuada $(10.06 \%)$ es la de "en clase, en presencia del tutor/a", lo que muestra el respeto evidente a esta figura por el grupo de alumnos/as que está bajo su responsabilidad. En la opción "en otros lugares" se aporta información sobre espacios externos a la escuela, como calles, 
plazas y parques de la localidad, donde se inician los conflictos o donde continúan los escolares.

Tabla 9.

Lugares donde se producen las acciones de acoso.

\begin{tabular}{lc}
\hline Lugares & Elecciones \\
\hline 1. En las entradas y salidas del colegio & $58.35 \%$ \\
2. En la clase, cuando está el tutor/a & $10.06 \%$ \\
3. En clase, cuando está otro maestro/a & $16.84 \%$ \\
4. En clase, cuando estamos solos, al cambiar el & $51.53 \%$ \\
maestro/a de grupo & $22.59 \%$ \\
5. En los pasillos & $18.00 \%$ \\
6. En los baños & $88.17 \%$ \\
7. En los recreos & $14.98 \%$ \\
8. En otros lugares & \\
\hline
\end{tabular}

Fuente: elaboración propia

\section{Intervenciones contra acoso}

Al igual que en el apartado anterior, la solicitud de datos sobre las intervenciones en el campo de la convivencia se realiza presentando un listado de opciones de las que se pueden señalar hasta tres opciones de aquellas que se llevan a cabo en el centro. En estas preguntas se proponen acciones de control, otras formativas y algunas de actitud del alumnado ante hechos de acoso referidas a la víctima y al agresor.

Se ha construido la tabla 10 con el porcentaje de alumnado que ha elegido cada una de las indicaciones propuestas.

De estas elecciones, se destacan:

- Los niños/as avisamos a los maestros/as (85.30\%).

- Los maestros/as castigan (83.30 \%).

- Los maestros/as nos llaman la atención (77.69\%).

- Los maestros/as nos hacen reflexionar en la clase y nos aconsejan $(75.56 \%)$.

- Los niños/as separamos las peleas (72.77\%).

Estas respuestas son destacadas por el alumnado como intervenciones más habituales. De ellas se puede desprender, en primer lugar, que el alumnado procura no implicarse cuando se están produciendo problemas relacionales y busca apoyo o protección en el profesorado; por otra parte, se hacen destacar las acciones punitivas con las llamadas de atención y los castigos; a continuación, se alude a la actividad formativa más común, la reflexión en el aula sobre las dificultades relacionales que se producen y recibir orientaciones para la mejora del clima de convivencia y, por último, paradójicamente la primera de las observaciones, se muestra una actitud positiva de intervención en los conflictos del alumnado que separa las peleas. 
Tabla 10

Intervenciones.

\begin{tabular}{lc}
\hline Intervenciones & Elecciones \\
\hline 1. Hay niños y niñas mediadores/as de la paz & $38.59 \%$ \\
2. Se dan charlas sobre la convivencia & $52.88 \%$ \\
3. En las clases/en las asambleas tratamos temas de & $50.19 \%$ \\
convivencia con los maestros/as & $77.69 \%$ \\
4. Los maestros/as nos llaman la atención & $75.56 \%$ \\
5. Los maestros/as nos hacen reflexionar en la clase y nos & $83.30 \%$ \\
aconsejan & $51.86 \%$ \\
6. Los maestros/as castigan & $63.27 \%$ \\
7. Los maestros/as "ponen partes" & $62.34 \%$ \\
8. Los maestros/as mandan a los alumnos/as al Director/a o al & $72.77 \%$ \\
Jefe/a de Estudios & $33.21 \%$ \\
9. Los niños/as ayudamos a la víctima & $85.30 \%$ \\
10. Los niños/as separamos las peleas & $12.24 \%$ \\
12. Los niños/as reñimos a los agresores & $58.49 \%$ \\
13. Me meto también en la pelea & $43.27 \%$ \\
14. Los maestros/as llaman a los padres & $7.88 \%$ \\
15. Se expulsa a los niños/as que son acosadores/as & 16. No se hace nada \\
\hline
\end{tabular}

Fuente: elaboración propia

Un segundo agrupamiento se puede realizar con las acciones que han sido elegidas por un $50 \%$ de los escolares y que reúne las siguientes propuestas (ver tabla 10):

- Los maestros/as mandan a los alumnos/as al Director/a o al Jefe/a de Estudios (63.27\%).

- Los niños/as ayudamos a la víctima (62.34\%).

- Los maestros/as llaman a los padres (58.49\%).

- Se dan charlas sobre la convivencia (52.88\%).

- Los maestros/as "ponen partes" (51.86\%).

- En las clases/en las asambleas tratamos temas de convivencia con los maestros/as (50.19\%).

Como se puede observar, persiste en este grupo de intervenciones la mezcla de acciones represivas, de implicación del alumnado y formativas, aunque no se destacan, precisamente, estas últimas.

Se puede establecer un tercer grupo, representado por las respuestas a los items:

- Se expulsa a los niños/as que son acosadores/as (43.27\%),

- Hay niños y niñas mediadores/as de la paz (38.59\%).

- Los niños/as reñimos a los agresores (33.21\%).

Estos vuelven a reunir las tres direcciones de la intervención referidas en los apartados anteriores, lo que muestra que la mediación está promovida por el centro y se le considera intervención formativa.

Las últimas propuestas elegidas, "Me meto también en la pelea" (que alcanza el $12.24 \%$ ) y "No se hace nada" (que obtiene el $7.88 \%$ ) deja constancia del rechazo del alumnado, aunque exista, a incitar a la agresión y a la presencia de intervención, mayoritariamente, en los centros escolares sobre el acoso escolar. 
Se concluye el apartado con la incorporación del siguiente gráfico (gráfico 1) en el que se visualizan los grupos de intervenciones comentados:

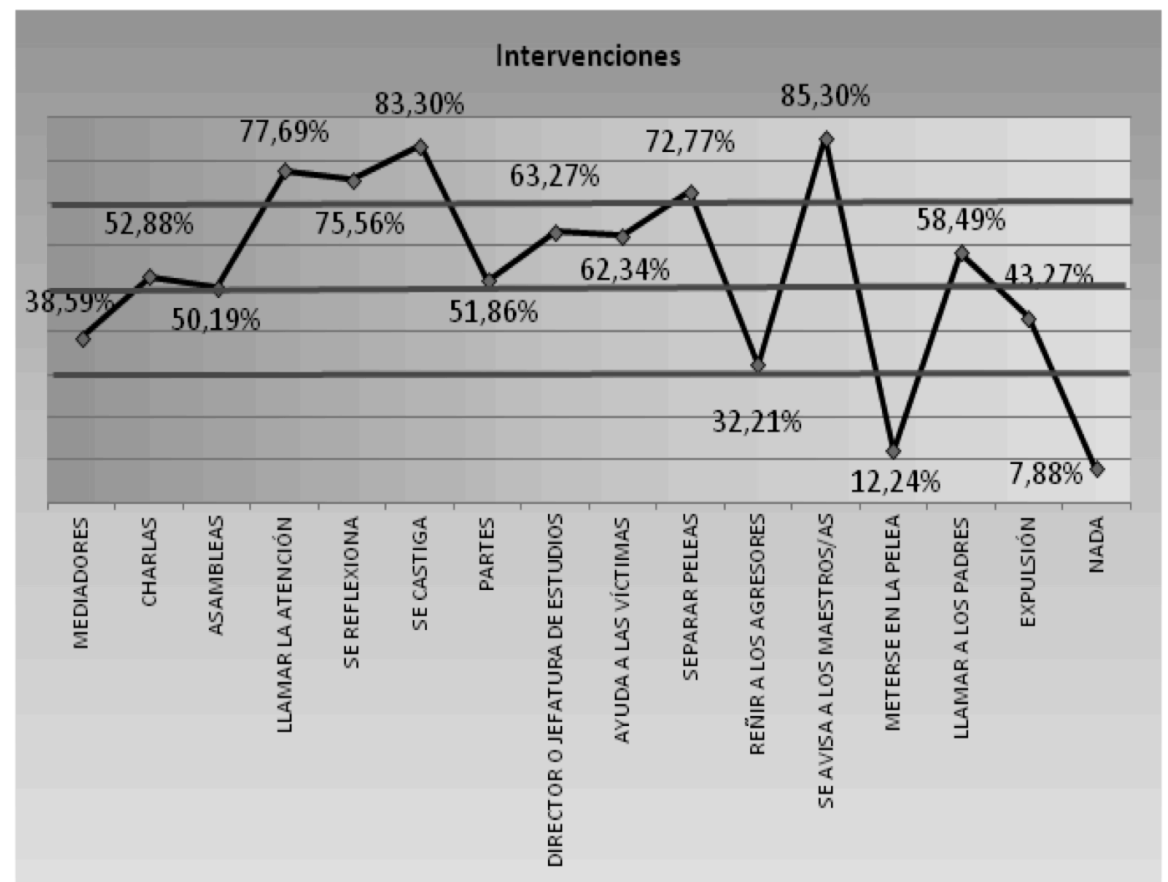

Gráfico 1. Intervenciones. Fuente: elaboración propia

Se han establecido lineas divisorias de áreas para visualizar más fácilmente los agrupamientos a los que se ha aludido en los anteriores comentarios: un tramo inicial hasta el $30 \%$; dos tramos intermedios de 20 puntos cada uno y un tramo final a partir del $70 \%$.

\section{COMENTARIOS}

En este artículo se ofrecen los resultados obtenidos del estudio realizado para valorar la percepción que tienen los niños y niñas sobre el maltrato entre compañeros y compañeras en los centros de Educación Primaria en la Provincia de Huelva. Atendiendo al primer objetivo específico planteado, "Valorar la percepción que tienen los niños/as sobre el acoso escolar en los centros de Educación Primaria en la Provincia de Huelva (España)", se puede concluir que las agresiones que se producen en sus distintas manifestaciones - Maltrato físico (directo, indirecto); Maltrato verbal (directo, indirecto); Exclusión social y Maltrato mixto- en términos generales, responden a un porcentaje mínimo de quienes las sufren, aunque este hecho no quita gravedad al asunto. Un número considerable de escolares se ven implicados en alguna ocasión en ellas como espectadores, víctimas o agresores, datos que concuerdan con estudios previos llevados a cabo sobre este tema (Alsaker \& Vilén, 2010; Cerezo, 2006).

Apoyando los resultados de diversas investigaciones (Albaladejo-Bázquéz et al., 2013; Carretero-Bermejo, 2011; Martínez, 2008; Ruiz, Riuró, \& Tesouro, 2015; Sánchez \& Ortega, 2010) se ofrecen las siguientes conclusiones: 
- Desde la percepción del alumnado, se entiende que las relaciones entre compañeros son buenas o muy buenas, aunque se originan hechos contrarios a las normas de convivencia.

- Sobre el ambiente de los centros escolares, la sensación de bienestar personal en el centro es superior en comparación con la observación que se realiza del ambiente del colegio.

- Se considera que la sensación de victimización en las tres intensidades establecidas (leve, moderada y grave) es alta.

- Destaca la presencia, en primer lugar, del maltrato verbal, tanto directo como indirecto, seguido de la exclusión social y el maltrato físico directo.

- El alumnado de Educación Primaria se define, generalmente, como maltratador ocasional; es baja su apreciación en los valores moderado y grave.

- En la intensidad grave, la actividad agresora se inclina por la exclusión, el maltrato verbal y el maltrato físico.

- Es más habitual que sean niños acosadores que forman grupos los autores de la actividad agresora; le siguen en elección los grupos de niños y niñas y, a continuación, un niño; en menor medida son elegidas las niñas, individualmente o en grupo.

- Los agresores se encuentran, regularmente, en una clase de niños mayores que la víctima, en la propia clase o en una clase del mismo nivel.

- Como espectadores se alcanzan los datos más altos sobre la presencia de intimidación en la escuela.

- Se establecen dos comportamientos en el alumnado: participar activamente de forma positiva -mediando en el conflicto-, o de forma negativa -incentivando las agresiones- y, por otra parte, pasivamente, como meros espectadores, sin realizar ningún tipo de acción.

- El alumnado procura no implicarse en los problemas relacionales.

- Se observan como acciones graves el maltrato verbal directo, el maltrato físico y el maltrato verbal indirecto.

- El lugar más común en el que se producen los acontecimientos de acoso escolar es el patio de recreo (Cerezo, 2006), de forma destacada; siguen en importancia las entradas y salidas, y en los cambios de clase.

Respecto al segundo objetivo, "Dar cuenta, desde la opinión del alumnado, de las intervenciones que se llevan a cabo en los centros escolares para solucionar los problemas de acoso escolar", se destacan las acciones punitivas sobre la actividad formativa.

En general, son los niños quienes presentan más capacidad de intervención en las situaciones de conflictividad, cuando estas se están produciendo.

En las intervenciones, los pequeños destacan las formativas y de ayuda, mientras que los mayores las disciplinarias.

Sobre el comportamiento de los docentes, el alumnado expresa una doble actitud de estos: los que se preocupan y los que no muestran interés por estos asuntos; sin embargo, el alumnado acude a los docentes para comunicar los problemas de acoso (Diaz-Aguado, Martínez-Arias, \& Martín-Babarro, 2004).

Este trabajo puede ser útil para establecer las líneas base en la elaboración de un proyecto de intervención orientadora que persiga la 
formación socializadora del alumnado. La realización de un proyecto de intervención para la prevención y la intervención del acoso escolar debe considerarse como un modelo abierto, adaptable necesariamente al contexto y a las dificultades expresadas en la evaluación inicial y permanentemente acomodada a la evolución del centro en el que se desarrolle. Respecto a las limitaciones del estudio, los resultados obtenidos quedan exclusivos a un contexto local y a la propia percepción del alumnado, en futuras investigaciones se podria contrastar estos resultados con otros sujetos implicados como pueden ser familiares y profesores y ampliar el tamaño de la muestra a otras localidades.

\section{REFERENCIAS}

Alsaker, F. D. \& Vilén, U. L. (2010). El bullying en la edad preescolar. En R. Ortega (Ed.), Agresividad injustificada, bullying y violencia escolar (pp. 129-164). Madrid: Alianza Editorial.

Albaladejo-Blázquez, N., Ferrer-Cascales, R., Reig-Ferrer, A., \& Fernández-Pascual, M.D. (2013). ¿Existe Violencia Escolar en Educación Infantil y Primaria? Una propuesta para su evaluación y gestión. Anales de psicologia, 3(29), 1060-1069. https://doi.org/10.6018/analesps.29.3.158431

Arón, A. (2008). Un programa de educación para la no violencia. En C. Berger \& C. Lisboa (Eds.), Violencia escolar: Estudios y posibilidades de intervención en Latinoamérica (pp. 265-296). Santiago, Chile: Editorial Universitaria.

Arroyave. P. (2012). Factores de vulnerabilidad y riesgo asociados al bullying. Revista CES Psicología, 5(1), $116-125$.

Avilés-Martínez, J. M. (1999). CIMEI. Cuestionario sobre intimidación y maltrato entre iguales. Valladolid: Autor.

Belsey, B. (2005). Ciberbullying: an emerging threta to the always of generation. Recuperado de http://www.cyberbullying.ca

Carbonell, J. L. (2009). Prevención de la violencia escolar. En J. Peredes \& A. de la Herrán (Eds.), La práctica de la innovación educativa, (pp. 101-119). Madrid: Síntesis.

Carretero-Bermejo, R. (2011). Sexo, sexismo y acoso escolar entre iguales. Revista complutense de educación, 22(1), 27-43. https://doi.org/10.5209/rev_RCED.2011.v22.n1.2

Cerezo, F. (2006). Análisis comparativo de variables socioafectivas diferenciales entre los implicados en el bullying. Estudio de un caso de víctima-provocador. Anuario de Psicología Clínica y de la Salud, 2, 2734.

Collell, J. \& Escudé, C. (2003). Cuestionario para mejorar el conocimiento sobre uno mismo y sobre las relaciones con los demás. Recuperado de http://www.amnistiacatalunya.org/edu/material/webquest/local/bullyingresadir-4cuestionarioes.pdf

Defensor del Pueblo (2000). Violencia Escolar: El Maltrato entre Iguales en la Educación Secundaria Obligatoria. Madrid: Defensor del Pueblo.

Diaz-Aguado, M. J., Martínez-Arias, R., \& Martín-Babarro, J. (2004). Prevención de la violencia y lucha contra la exclusión desde la adolescencia. Madrid: Ministerio de Trabajo y Asuntos Sociales, INJUVE.

Díaz-Aguado, M.J., Martínez-Arias, R., \& Martín-Babarro, J. (2013). El acoso entre adolescentes en España. Prevalencia, papeles adoptados por todo el grupo y características a las que atribuyen la victimización. Revista de Educación, 362, 348-379.

Farrington, D. (2005). Childhood origins of antisocial Behavior. Clinical Psychology and Psychotherapy, 12, 177-190. https://doi.org/10.1002/cpp.448

Fernández, I. \& Ortega, R. (1998). Cuestionario sobre abusos entre compañeros. En I. Fernández (Ed.), Prevención de la violencia y resolución de conflictos. El clima escolar como factor de calidad, (pp. 210214). Madrid: Narcea.

Gómez-Bahillo, C. (2006). Protocolo para la medición de la convivencia en los Centros educativos no universitarios de la Comunidad autónoma de Aragón. Cuestionario para las asociaciones de madres y padres de alumnos. Recuperado https://convivencia.files.wordpress.com/2012/05/investigacion_convivencia_aragong_bahillo2006218p.pdf

Jiménez, A. (2007). El maltrato entre escolares (bullying) en el primer ciclo de educación secundaria obligatoria: valoración de una intervención a través de medios audiovisuales. (Tesis inédita de doctorado). Universidad de Huelva, Huelva.

Lera, M. J. \& Olías, F. (2002). Cuestionario de nominaciones Sociograma. Recuperado de http://www.psicoeducacion.eu/files/bullying/Instrumentos_bullying.pdf

Marín-Martínez, A. \& Reidl-Martínez, L.M. (2013). Validación Psicométrica Del Cuestionario "Así Nos Llevamos En La Escuela" Para Evaluar El Hostigamiento Escolar (Bullying) En Primarias. Revista Mexicana de Investigación Educativa, 18(56), 11-36.

Martínez, A. (2008). Situaciones de violencia en centros escolares. Madrid: FETE-UGT.

Martinez, P. (2012). El maltrato entre iguales en Educación Primaria y ESO. Bilbao: Instituto Vasco de Evaluación e Investigación Educativa.

Olweus, D. (2006). Conductas de acoso y amenaza entre escolares $\left(3^{a}\right.$ Ed.). Madrid: Ed. Morata. 
Ortega, R., del Rey, R., \& Mora-Merchán, J. (2001). Violencia entre escolares: conceptos y etiquetas verbales que definen el fenómeno del maltrato entre iguales. Revista Interuniversitaria de Formación del Profesorado, 41, 95-113.

Ortega, R., del Rey, R., \& Mora-Merchán, J. A. (2008). Cuestionario sobre Convivencia, Conflictos y Violencia Escolar. Cuestionario del alumnado de Primaria. Recuperado de http://www.uco.es/laecovi/img/recursos/Niqo6GT1bRS2R0u.pdf

Ovejero, A., Smith, P. K., \& Yubero, S. (2013). El acoso escolar y su prevención. Perspectivas internacionales. Madrid: Biblioteca Nueva.

Quintana P. A., Montgomery U. W., Malaver S. C., \& Ruiz S. G. (2010). Capacidad de disfrute y percepción del apoyo comunitario en adolescentes espectadores de episodios de violencia entre pares (Bullying). Revista de Investigación en Psicologia, 13(1), 139-150. https://doi.org/10.15381/rinvp.v13i1.3742

Rodicio-García, M. L. \& Iglesias-Cortizas, M. J. (2011). El acoso escolar. Diagnóstico y Prevención. Madrid: Biblioteca Nueva.

Ruiz, R., Riuró, M., \& Tesouro, M. (2015). Estudio del bullying en el ciclo superior de primaria. Educación $X X 1,18(1), 345-368$.

Sánchez, V. \& Ortega, R. (2010). El estudio científico del fenómeno bullying. En R. Ortega (Coord.), Agresividad injustificada, bullying y violencia escolar, (pp. 55-80). Madrid: Alianza Editorial.

Wang, J., Iannotti, R. J., \& Nansel, T. R. (2009). School bullying Among Adolescents in the United States: physical, verbal, relational and cyber. Journal of Adolescent Health, 45, 368-375. https://doi.org/10.1016/j.jadohealth.2009.03.021

Recibido 11-12-2017 | Aceptado 08-03-2018

Este trabajo se encuentra bajo una Licencia Creative Commons Atribución 4.0 Internacional que permite a terceros utilizar lo publicado siempre que se dé el crédito pertinente a los autores y a Psicodebate 